\title{
Mise en évidence d'une activité lipoprotéine-lipasique dans le tissu adipeux de chèvre : comparaison de trois méthodes d'extraction
}

par Y. CHILLIARD, Michelle DORLEANS et P. M. FEHR.

\author{
Laboratoire de recherches de la chaire de zootechnie (I.N.R.A.) \\ Institut national agronomique Paris-Grignon \\ 16, rue Claude-Bernard 75231 Paris Cedex 05
}

Summary. Lipoprotein lipase activity in goat adipose tissue : comparison of three extraction methods.

Lipoprotein lipase (L.P.L.) activity is studied in dairy goat omental adipose tissue and three methods of extracting it are compared.

1) in vitro incubation of tissue pieces in a medium containing heparin and goat serum (I.V. method).

2) homogenization in an ammoniacal buffer containing heparin (H.A. method).

3) homogenization of acetone-ether defatted powders in the ammoniacal buffer (E.A. method).

These three types of crude extracts show lipolytic activity on « activated » Intralipid triglycerides. The effects of inhibitors and activators indicate that these activities are essentially due to enzyme L.P.L. Lipolytic activity is inhibited by an excess of substrate and has an apparent $\mathrm{Km}$ of $0.19 \mathrm{mM}$ of the substrate triglyceride.

Reproductibility of the successive steps of L.P.L. activity assay is estimated. Extraction by in vitro tissue incubation (I.V. method) is less reproducible than the two other types of extraction (H.A. and E.A. methods).

L.P.L. activity obtained by the 3 extraction methods is similar and highly correlated in the 13 tissue samples from 6 goats in different physiological states.

\section{Introduction.}

Le rôle de l'enzyme lipoprotéine-lipase (L.P.L.) dans le prélèvement des acides gras des iriglycérides des chylomicrons et des lipoprotéines sanguins par le tissu adipeux a été mis en évidence entre autres par Bezman et al. (1962), Robinson (1963), Garfinkel et al. (1967), Scow et al. (1972). L'activité L.P.L. du tissu adipeux a été étudiée essentiellement par trois méthodes : extraction de la L.P.L. à partir de poudres acéto- 
niques (Korn et Quigley, 1955) puis acéło-éthérées du tissu (Robinson, 1963), élution de la L.P.L. par incubation du tissu en milieu hépariné (Cherkes et Gordon, 1959), préparation d'homogénats aqueux du tissu adipeux (Korn et Quigley, 1955).

D'après Benson (1969), la L.P.L. du tissu adipeux de ruminant présente des propriétés particulières. D'une part, cette L.P.L. n'est pas éluée lors d'incubations du tissu adipeux de vache en milieu hépariné ; d'autre part, l'héparine ne stimule pas l'activité lipolytique présente dans les homogénats aqueux du tissu. Toutefois, Gill (1970) observe, chez la chèvre, la présence d'une activité L.P.L. dans le plasma posthéparine. N'ayant pas obtenu de résultats satisfaisants en utilisant des poudres acétoéthérées de tissu adipeux, à la différence de Haugeback ef al. (1974) avec des tissus d'agneaux, Benson (1969) a préconisé l'utilisation de la méthode des homogénats aqueux pour l'étude de l'activité L.P.L. du tissu adipeux de vache.

Compte tenu de la diversité des méthodes utilisées pour l'extraction de l'activité L.P.L. des tissus adipeux, et des problèmes spécifiques que semblent poser les tissus adipeux de ruminant, il est apparu nécessaire de comparer de façon systématique les trois méthodes généralement utilisées : incubation du tissu in vitro (I.V.), homogénats aqueux (H.A.) et extraction à partir des poudres acéto-éthérées (E.A.), après les avoir adaptées au tissu adipeux de chèvre.

\section{Matériel et méthodes.}

Les principaux produits ou appareils utilisés sont les suivants :

- Xylazine (chlorhydrate de 2-(2,6-xylidino)-5, 6-dihydro-4H-1,3-thiazine ; Bay Va 1470 ; Rompun ; Bayer) ;

- Albumine bovine (Cohn fraction V-Powder ; Sigma) ;

- Acetone (R. P. Normapur ; Prolabo) ;

- Ether diethylique (R. P. Normapur ; Prolabo) ;

- Intralipide 10 p. 100 (Vitrum) ;

- Ommi-mixer Sorvall, muni d'un microhomogénéiseur ;

- Appareil de filtration en verre, de diamètre $10 \mathrm{~cm}$ (Sartorius).

Les solutions sont préparées comme suit :

- Le tampon Krebs-Ringer-Bicarbonate selon la méthode décrite par Cohen (1949) ;

- Le tampon ammoniacal hépariné à partir d'une solution de chlorure d'ammonium (50 $\mathrm{mM}$ ) contenant 5,6 U.I./ml d'héparinate de sodium, ajustée à $\mathrm{pH} 8,3$ (à $20^{\circ} \mathrm{C}$ ) avec une solution normale d'ammoniaque.

\section{Description des méthodes utilisées}

Six chèvres laitières de race Alpine âgées de 3 à 7 ans, cinq en fin de gestation puis en début de lactation ef une chèvre sèche non gestante, reçoivent une ration à base de foin de luzerne distribué à volonté, et d'aliments concentrés (orge, avoine, tourteau de soja, minéraux, vitamines). 


\section{A. Biopsies de tissu adipeux épiploïque.}

Les chèvres reçoivent 0,5 à $1 \mathrm{mg} / \mathrm{kg}$ de Xylazine (sédatif et analgésique) par voie intraveineuse, le matin alors qu'elles n'ont pas reçu d'aliments depuis $12 \mathrm{~h}$ environ. Après infiltration sous cutanée de Procaine, une laparotomie permet de prélever rapidement 20 à $50 \mathrm{~g}$ de grand épiploon (Omentum majus). Le tissu est conservé à $-25^{\circ} \mathrm{C}$.

B. Méthodes d'extraction de l'activité L.P.L. du tissu adipeux.

1. Par incubation in vitro (méthode I.V.).

Des morceaux de tissu (de moins de 3 à $4 \mathrm{mg}$ ) sont découpés, pesés puis incubés à $35^{\circ} \mathrm{C}$ pendant une heure sous agitation dans $13 \mathrm{ml} / \mathrm{g}$ de tampon Krebs-RingerBicarbonate à $\mathrm{pH} 7,4$, contenant 1,1 p. $100(\mathrm{p} / \mathrm{v})$ d'albumine bovine, 4 p. $100(\mathrm{v} / \mathrm{v})$ de sérum frais de chèvre et $6,4 \mathrm{U} .1 . / \mathrm{ml}$ d'héparinate de sodium. Le milieu d'incubation est récupéré par filtration et conservé à $0^{\circ} \mathrm{C}$ jusqu'à l'essai de l'activité enzymatique, dans les deux heures qui suivent.

\section{Par homogénéisation en milieu aqueux (méthode H.A.).}

La technique adoptée est proche de celle utilisée par Benson (1969). Cependant l'homogénéisation est réalisée dans le tampon ammoniacal hépariné (de Gasquet et Péquignot, 1972).

Environ $600 \mathrm{mg}$ d'épiploon prédécoupé sont broyés ( 2 fois 10 secondes à $50000 \mathrm{tr} /$ $\mathrm{mn}$ ) dans $4 \mathrm{ml}$ de tampon ammoniacal hépariné à $0{ }^{\circ} \mathrm{C}$. Le broyat est alors passé dans un homogénéiseur de Potter à $0-4^{\circ} \mathrm{C}$ puis le liquide surnageant est filtré. Le culot gras restant dans le Potter est soumis à une $2^{\mathrm{e}}$ extraction dans les mêmes conditions.

3. A partir de poudres acéto-éthérées (méthode E.A.).

La méthode de dégraissage de Hamosh et al. (1970) a été modifiée, en raison de l'utilisation de quantités relativement importantes de tissus contenant des graisses à point de fusion élevé.

L'épiploon prédécoupé (750 à $1500 \mathrm{mg}$ ) et $100 \mathrm{mg}$ d'albumine bovine sont homogénéisés dans $50 \mathrm{ml}$ d'acétone à $0^{\circ} \mathrm{C}$ puis transvasés dans un erlenmeyer avec environ un litre d'acétone. Après agitation, ce mélange est filtré sur l'appareil Sartorius muni d'un papier Whatman $n^{\circ} 50$. Le dégraissage est complété en versant sur le précipité un litre environ d'éther diéthylique. Les poudres sèches et dégraissées sont aussitôt récupérées et conservées quelques jours à $-25^{\circ} \mathrm{C}$ sans perte d'activité décelable.

Une à deux heures avant l'essai de l'activité enzymatique, les poudres acétoéthérées sont agitées quelques minutes dans 10 à $20 \mathrm{ml}$ de tampon ammoniacal hépariné à $0{ }^{\circ} \mathrm{C}$ puis homogénéisées dans un « polybroyeur de tissus ».

\section{Essai de l'activité L.P.L. des extraits bruts.}

Le substrat est obtenu en « activant 》 une émulsion commerciale (Intralipide à 10 p. 100) au cours d'une incubation ( $45 \mathrm{mn}$ à $35^{\circ} \mathrm{C}$, sous agitation) en présence de sérum frais de chèvre, toujours prélevé sur un même animal qui n'a pas subi de biopsie. 
Le rapport $8 \mathrm{ml}$ de sérum pour $100 \mathrm{mg}$ de triglycérides de l'Intralipide, préconisé par Robinson (1963), a été adopté. Il correspond à la stimulation maximum de l'activité L.P.L. du lait de chèvre (Chilliard ef Fehr, 1976).

L'incubation enzymatique $\left(1 \mathrm{~h}\right.$ à $35^{\circ} \mathrm{C}$ sous agitation) débute à l'instant où $1 \mathrm{ml}$ d'extrait enzymatique brut est ajouté à $1,4 \mathrm{ml}$ du mélange suivant : 3 vol. d'Intralipide activé ; 3 vol. d'une solution à $18 \mathrm{p}$. $100(\mathrm{p} / \mathrm{v})$ d'albumine bovine dans $\mathrm{NaCl} 0,154 \mathrm{M}$, à $\mathrm{pH} 8,3 ; 1$ vol. de tampon Tris- $\mathrm{HCl}, 1,6 \mathrm{M}$, à pH 8,3.

Chaque extrait enzymatique est incubé en double, et les concentrations en acides gras non estérifiés (A.G.N.E.) sont mesurées, aux temps zéro et soixante minutes, par la méthode semi-automatique d'Antonis (1965). Les lipides sont extraits par l'éther di-iso-propylique en présence d'acide silicique, qui adsorbe les phospholipides, puis sont repris par le chloroforme. Les extraits chloroformiques sont passés à l'autoanalyseur (Technicon-A II) et les A.G.N.E. sont dosés sous forme de savons cuivriques formant un complexe coloré avec le diéthyldithiocarbamate de sodium. L'activité L.P.L. est exprimée en $\mu$ Eq d'acides gras libérés par heure et par gramme de tissu.

\section{Contrôle de la validité des méthodes utilisées}

\section{A. Méthode de biopsie.}

Nous avons observé que la Xylazine n'interfère pas à court terme avec l'activité L.P.L. du tissu adipeux, au cours d'essais réalisés in vivo sur lapins de la même portée ayant, ou non, reçu de la Xylazine et sur une chèvre dont le prélèvement d'épiploon était fait avec ou sans Xylazine, ainsi qu'in vitro par incubation du tissu adipeux de chèvre dans un milieu hépariné contenant de la Xylazine, ou par addition de ce produit lors de l'essai de l'activité L.P.L.

\section{B. Méthode de dosage des A.G.N.E.}

La validité de la méthode d'Antonis (1965) pour le dosage des A.G.N.E. dans un système de mesure de la L.P.L. a été vérifiée en la comparant avec la méthode colorimétrique de Novak (1965) basée sur l'extraction des lipides selon Dole (1956) (Chilliard et Luzeau, résultats non publiés).

La reproductibilité de la phase finale de ce dosage a été estimée en passant successivement deux parties aliquoles de tous les extraits chloroformiques utilisés dans cette étude, à l'autoanalyseur. Les 779 couples de valeur (1er passage-2e passage) ainsi obłenus ont été analysés par régression linéaire. La valeur du coefficient de corrélation associé à la droite de régression est de 0,995. L'écart-type résiduel de $0,932 \mu \mathrm{Eq} / /$ et le coefficient de variation résiduelle de 3,23 p. 100 fournissent une estimation de la reproductibilité de cette étape du dosage (Snedecor et Cochran, 1957).

La reproductibilité de l'ensemble du dosage des A.G.N.E. a été estimée en dosant des milieux d'incubation " au temps zéro » (immédiatement après addition de l'extrait enzymatique). Pour des concentrations en A.G.N.E. d'environ $400 \mu \mathrm{Eq} / \mathrm{l}$, les coefficients de variations obtenus au cours de deux expériences sont de 2,6 p. $100(n=20)$ ou 
de 4,1 p. 100 ( $n=14$ ) (chacun des 34 résultats étant lui-même la moyenne des résultats de deux passages à l'autoanalyseur).

C. Contrôle des blancs sans extrait enzymatique.

II n'y avait pas de libération d'A.G.N.E. dans le milieu incubé pendant une heure en absence d'extrait enzymatique.

\section{Résultats.}

\section{Etudes préliminaires}

\section{A. Concentration en A.G.N.E. des extraits bruts.}

Le milieu d'incubation au temps zéro contient des A.G.N.E. apportés par l'Intralipide, le sérum de chèvre et l'albumine bovine (qui sont retrouvés dans le blanc sans extrait enzymatique), ainsi que par les extraits bruts.

Les extraits bruts obtenus par les méthodes H.A. ef E.A. contiennent des quantités très faibles et très peu variables d'A.G.N.E. Par contre, avec la méthode I.V., les teneurs en A.G.N.E. des extraits bruts sont élevées, variables pour un même tissu et différentes d'un tissu à l'autre lorsque les extraits ont des activités lipolytiques inégales.

B. Linéarité de l'activité lipolytique.

Les libérations d'A.G.N.E. sont proportionnelles aux concentrations en extraits bruts ajoutés $(0,25$ à 1,5 ml) et au temps d'incubation (15 à $75 \mathrm{mn}$ ).

C. Variabilité des résultats de l'incubation enzymatique (doubles d'incubation).

Pour 163 extraits enzymatiques obtenus à partir de 13 tissus adipeux par les 3 méthodes, les activités L.P.L. des doubles d'incubation ont été analysées par régression linéaire. Le coefficient de corrélation associé à la droite de régression est de 0,984 . L'écart-łype résiduel de $1,01 \mu \mathrm{Eq} / \mathrm{h} / \mathrm{g}$, et le coefficient de variation résiduelle de 12 p. 100 , fournissent une estimation de la reproductibilité de l'incubation.

\section{Caractérisation de l'activité lipolytique enregistrée}

A. Influence des facteurs inhibant spécifiquement la L.P.L.

L'activité lipolytique de l'épiploon d'une chèvre gestante (environ $15 \mu \mathrm{Eq} / \mathrm{h} / \mathrm{g}$ ) est peu inhibée, quel que soit le type d'extrait, par le fluorure de sodium (-12 à - $20 \mathrm{p}$. 100) (fig. 1). L'activité est fortement inhibée (-73 à - 95 p. 100) par le chlorure de sodium $(0,5 \mathrm{M})$. Par contre, alors que les extraits obtenus por les méthodes H.A. et E.A. sont très peu actifs sur l'Intralipide non activé ( 5 à 7 p. 100 de l'activité avec le substrat activé), l'extrait obtenu par la méthode I.V., qui contient du sérum, conserve 55 p. 100 de son activité.

Afin de déceler une éventuelle interférence d'activités lipolyłiques du tissu impli- 
quées dans la libération des acides gras des triglycérides stockés dans l'adipocyte, une expérience d'inhibition par le chlorure de sodium $(0,5 M)$ a été effectuée sur l'épiploon de la même chèvre, prélevé en début de lactation (activité d'environ $5 \mu \mathrm{Eq} /$ $\mathrm{h} / \mathrm{g}$ ). Cette chèvre produisait une quantité importante de lait $(2,8 \mathrm{~kg} / \mathrm{j})$ et présentait des signes de mobilisation des triglycérides de réserves (teneurs élevées en A.G.N.E. et en $d$ - $\beta$-hydroxybutyrate dans le plasma sanguin). Les activités lipolytiques des trois types d'extraits sont très fortement inhibées par $\mathrm{NaCl} 0,5 \mathrm{M}(-94$ à -100 p. 100). L'activité lipolytique observée en début de lactation présente donc les mêmes caractéristiques d'inhibition par le chlorure de sodium $(0,5 \mathrm{M})$ que celle observée en fin de gestation.

\section{B. Influence de l'héparine.}

L'héparine interagił avec la L.P.L. du tissu adipeux (Korn et Quigley, 1955 ; Korn, 1962) et son effet a été testé sur l'extraction de l'enzyme ainsi que sur l'activité L.P.L., une fois que celle-ci est extraite en absence d'héparine (fig. 2), à partir d'un

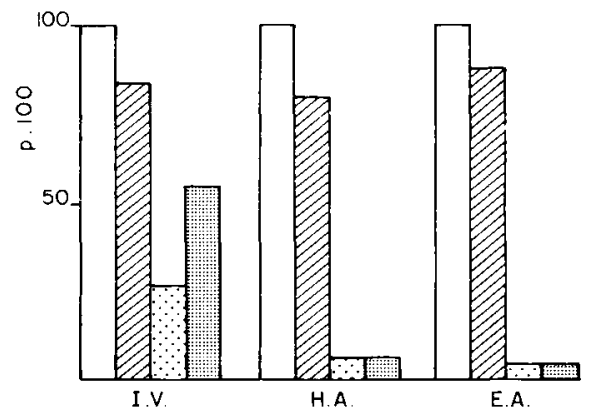

FIG. 1. - Influence du fluorure de sodium, du chlorure de sodium et de la non-activation du substrat sur l'activité lipolytique extraite par trois méthodes.

L'activité enregistrèe dans les conditions standard par chaque méthode est prise comme base 100 . Les concentrations finales en $\mathrm{NaF}$ QTZ et en $\mathrm{NaCl} \cdots$ sont $18 \mathrm{mM}$ et $0,52 \mathrm{M}$. Une solution de $\mathrm{NaCl} 0,154 \mathrm{M}$ remplace le sérum de chèvre dans le substrat non activé

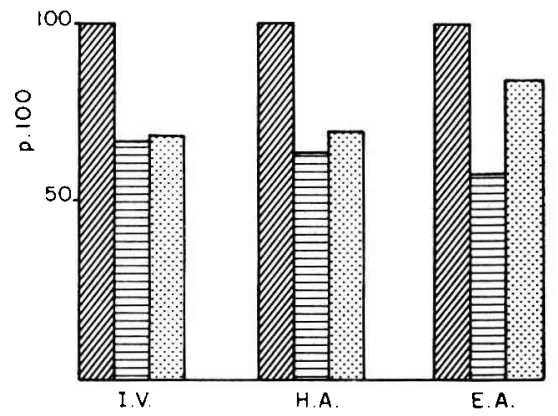

FIG. 2. - Influence de l'héparine sur l'activité lipolylique

L'héparine est soit ajoutée à l'extraction dans les conditions standard $\mathbb{Z}$; soit omise ; soit supprimée à l'extraction et ajoutée au milieu d'incubation de façon à obtenir la même concentration finale que dans les conditions standard

tissu de chèvre gestante. Alors que seulement 58 à 67 p. 100 de l'activité est extraite en absence d'héparine par les trois méthodes, seule l'activité des extraits brułs obtenus par la méthode E.A. en absence d'héparine est stimulée de façon importante par l'héparine lors de l'incubation (+ 44 p. 100).

Bensadoun ef al. (1974) ayant observé une très forte augmentation de l'efficacité d'extraction de la L.P.L. des poudres acéto-éthérées de tissu adipeux de porc par l'héparine concentrée $(200 \mathrm{U} .1 . / \mathrm{ml})$, trois variantes d'extraction ont été essayées : 1) homogénéisation des poudres acéto-éthérées dans les conditions standard (5,6 U.I. 
d'héparine par $\mathrm{ml}$ de tampon ammoniacal) ; 2) homogénéisation dans $1 \mathrm{ml}$ d'une solution aqueuse à $200 \mathrm{U} . \mathrm{I} . / \mathrm{ml}$ d'héparine pendant $1 \mathrm{~h}$ à $0{ }^{\circ} \mathrm{C}$, puis dilution dans du tampon ammoniacal sans héparine, de façon à obtenir une concentration de 13,3 U.I. d'héparine $/ \mathrm{ml} \mathrm{;3)} \mathrm{homogénéisation} \mathrm{dans} \mathrm{un} \mathrm{tampon} \mathrm{ammoniacal} \mathrm{contenant} \mathrm{13,3} \mathrm{U.I.}$ d'héparine/ml. Pour chaque modalité deux extraits ont été réalisés, chacun incubé en double. Les activités L.P.L. obtenues $(15,1-15,8-16,3 \mu \mathrm{Eq} / \mathrm{h} / \mathrm{g})$ ne diffèrent pas significativement au seuil 10 p. 100.

C. Influence de la concentration en substrat.

Les activités enzymatiques sont souvent déterminées en utilisant une concentration élevée en substrat, de façon à obtenir une vitesse proche de la vitesse maximum apparente.

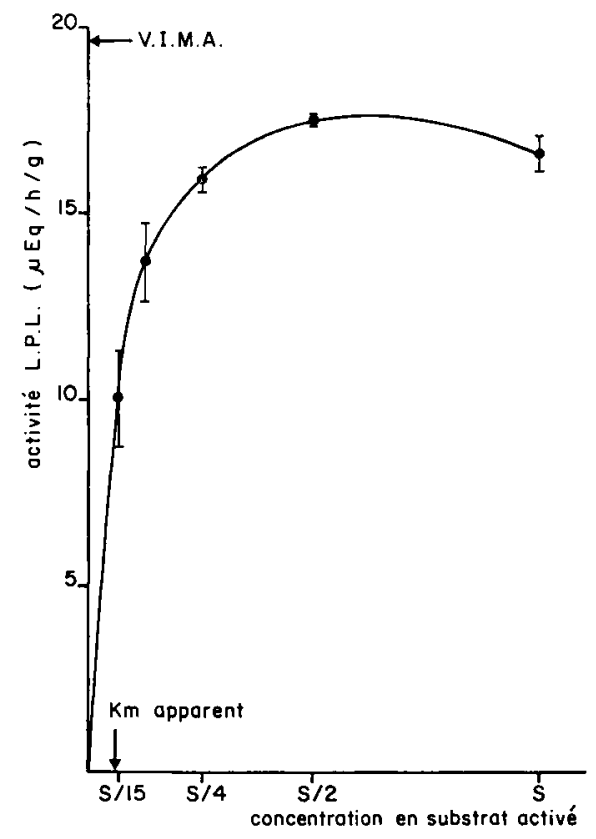

FIG. 3. - Influence de la concentration en substrat sur l'activité L.P.L.

(V.I.M.A. : vitesse initiale maximum apparente).

L'essai est réalisé avec un extrait brut obtenu par la méthode E.A., un temps d'incubation de $20 \mathrm{mn}$ ef des dilutions du substrat activé réalisées dans une solution de $\mathrm{NaCl} 0,154 \mathrm{M}$. La moyenne ( \pm écart-type) de 3 déterminations sont présentés. Les activités lipolytiques ont été calculées en utilisant la droite de régression des concentrations en A.G.N.E. au temps zéro pour chaque concentration en substrat $(r=0,98 ; n=10)$.

Avant d'étudier l'influence de la concentration en substrat, la période de vitesse initiale a été déterminée pour la plus faible concentration employée. Alors que la libération d'acides gras est linéaire pendant $60 \mathrm{mn}$ au moins avec la concentration 
standard en substrat (3,175 mM de triglycérides de l'Intralipide, activés), elle ne l'est que pendant $20 \mathrm{mn}$ avec une concentration quinze fcis plus faible.

D'après la figure 3 , il apparaît une légère inhibition de l'activité L.P.L. lorsque la concentration en substrat augmente entre 1,5 et $3 \mathrm{mM}$ de triglycérides de l'Intralipide, activés. Un $\mathrm{Km}$ apparent de $0,19 \mathrm{mM}$ de triglycérides de l'Intralipide, activés, et une vitesse initiale maximum apparente de $19,6 \mathrm{\mu Eq} / \mathrm{h} / \mathrm{g}$ ont été calculés, par la méthode de Eisenthal et Cornish-Bowden (1974), à partir des données correspondant aux quatre plus faibles concentrations en substrat.

\section{Comparaison des activités L.P.L. obtenues par les trois méthodes}

\section{A. Reproductibilité de l'extraction de l'activité L.P.L.}

A partir de chacun des 13 tissus éłudiés l'extraction de l'activité L.P.L. a été répétée au moins deux fois pour chacune des trois méthodes. Les résultats de reproductibilité intègrent les variabilités dues à l'hétérogénéité éventuelle des tissus, à l'extraction de l'enzyme, à la conservation, aux réactifs ef éventuellement à l'évolution du sérum de la chèvre donneuse.

Les variances intra-tissu, liées aux répétitions d'extraction par chaque méthode, ne sont pas significativement différentes au seuil 25 p. 100 entre les méthodes H.A. et

\section{TABLEAU I}

Analyse de la variance des activités L.P.L. des extraits bruts obtenus à partir de 13 tissus

\begin{tabular}{|c|c|c|c|}
\hline Méthode d'extraction & I.V. & H.A. & E.A. \\
\hline Nombre d'extraits $\ldots . \ldots \ldots \ldots \ldots \ldots \ldots$ & 34 & 35 & 49 \\
\hline Activité L.P.L. moyenne $(\mu \mathrm{Eq} / \mathrm{h} / \mathrm{g}) \ldots \ldots \ldots \ldots$ & 5,62 & 6,93 & 8,08 \\
\hline Signification de l'effet tissu $\ldots \ldots \ldots \ldots \ldots$ & $P<0,001$ & $P<0,001$ & $P<0,001$ \\
\hline Ecart-type résiduel (intra-tissu) $(\mu \mathrm{Eq} / \mathrm{h} / \mathrm{g}) \ldots$ & 2,34 & 1,59 & 1,41 \\
\hline Coefficient de variation résiduelle (p. 100) .. & 42 & 23 & 17 \\
\hline
\end{tabular}

L'activité L.P.L. moyenne des 13 tissus, calculée en pondérant d'après le nombre d'extraits réalisés par chaque méthode et pour chaque tissu est de 3,96 (I.V.) ; 4,29 (H.A.) ef 4,58 (E.A.) $\mu$ Eq/h/g.

E.A. Par contre, la variance intra-tissu observée avec la méthode I.V. diffère significativement (aux seuils 5 p. 100 et 1 p. 100) de celles des méthodes H.A. et E.A. (respectivement). Les coefficients de variation résiduelle que l'on peut calculer à partir de ces analyses (tabl. 1) sont apparemment élevés. Nous avons, en outre, calculé les paramètres de dispersion pour des séries d'extraits obtenus sur deux tissus à forte activité L.P.L. (†abl. 2). Les résultats des tableaux 1 et 2 sont très voisins puisque dans 
les deux cas la dispersion de la méthode I.V. est plus grande que celle des deux autres méthodes ; de plus, les écarts-types observés pour les 3 méthodes sont peu différents dans les deux tableaux.

TABLEAU 2

Reproductibilité de l'extraction de l'activifé L.P.L. de deux tissus

Méthodes d'extraction

I.V.

H.A.

E.A.

\begin{tabular}{lccccc}
\hline Tissu $\ldots \ldots \ldots \ldots \ldots \ldots \ldots \ldots \ldots$ & A & A & A & B \\
\hline Nombre d'extraits $\ldots \ldots \ldots \ldots \ldots \ldots$ & 9 & 11 & 15 & 7 \\
\hline Nombre de séries de mesures $\ldots \ldots$ & 5 & 7 & 7 & 4 \\
\hline Durée totale (semaines) $\ldots \ldots \ldots \ldots$ & 11 & 11 & 14 & 13 \\
\hline Activité L.P.L. moyenne $(\mu \mathrm{Eq} / \mathrm{h} / \mathrm{g}) \ldots$ & 11,9 & 14,2 & 15,9 & 10,9 \\
\hline Ecart-type $(\mu$ Eq/h/g) $\ldots \ldots \ldots \ldots \ldots$ & 3,48 & 2,18 & 1,79 & 0,90 \\
\hline Coefficient de variation $(\mathrm{p} .100) \ldots \ldots$ & 29 & 15 & 11 & 9 \\
\hline
\end{tabular}

B. Comparaison des activités absolues.

Les 39 résultats moyens obtenus par les 3 méthodes pour les 13 tissus ont été utilisés pour comparer les méthodes deux à deux par régression linéaire (fig. 4) (la transformation logarithmique des données ne change pas les tendances observées).
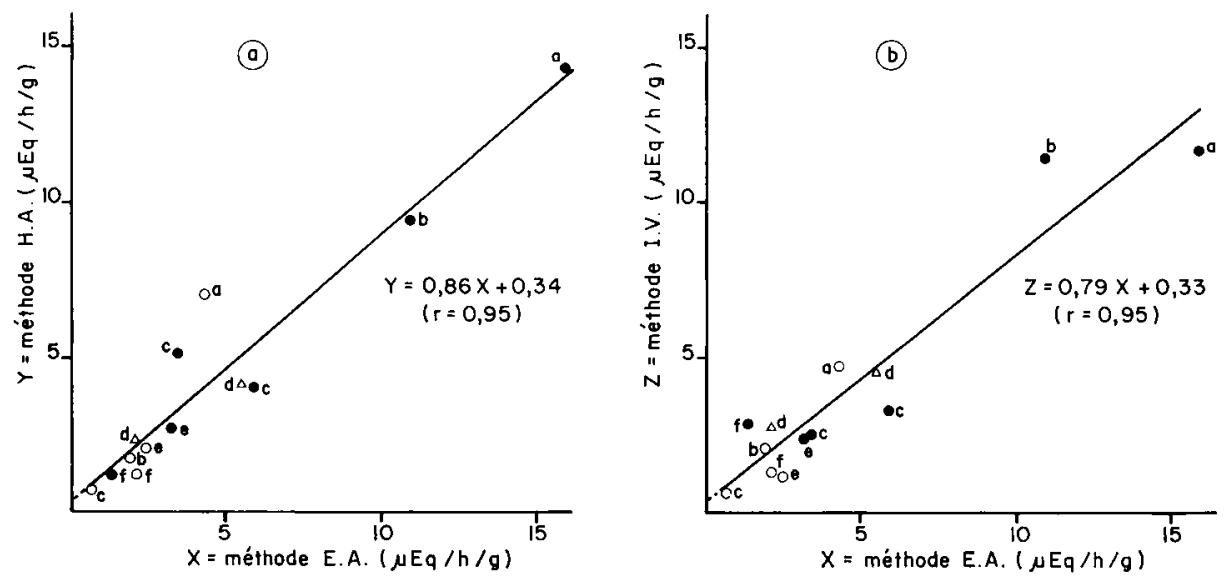

FIG. 4. - Liaison entre les activités L.P.L. moyennes observées pour un même tissu par les méthodes E. A., H.A. et I.V.

Chaque chèvre est représentée par une lettre. Les tissus ont été prélevés sur des femelles en fin de gestation (O), début de lactation $(O)$ ou sèche non-gestante $(\Delta)$. 
Les mesures obtenues par les méthodes H.A. et E.A. (fig. 4a) pour un même tiss sont très voisines puisque le coefficient de régression $(0,86)$ ne diffère pas significativement de 1 au seuil 10 p. 100 ef que l'interception avec l'axe des ordonnées $(+0,34)$ n'est pas significativement différente de zéro. Les variations observées avec la méthode H.A. sont donc pour 90 p. 100 les mêmes que celles de la méthode E.A. La comparaison entre les méthodes I.V. et E.A. (fig $4 b$ ) donne des résultats similaires. Cependant, le coefficient de régression $(0,79)$ est significativement différent de 1 au seuil 5 p. 100. La méthode I.V. semble donc donner des résultats inférieurs en valeur absolve à ceux obtenus avec la méthode E.A.

\section{Discussion.}

Nature de l'activité lipolytique observée.

La L.P.L. est faiblement inhibée par le fluorure de sodium (Cherkes €t Gordon, 1959) qui est un inhibiteur d'autres activités lipolytiques présentes dans le tissu adipeux (activité triglycéride-lipasique « hormono-sensible » (Rizack, 1961 ; Gorin et Shafrir, 1964) ; activités mono-, di- et triglycéride-lipasiques des extraits éthérés (Strand ef al., 1964).

La L.P.L. est très fortement inhibée par le chlorure de sodium 0,3 à $1 M$ (Korn, 1959) qui n'inhibe pas d'autres activités lipolytiques: activité triglycéride-lipasique " hormonosensible » (Rizack, 1961 ; Heller et Steinberg, 1972) ; activités monoglycéride-lipasiques (Biale et Shafrir, 1969 ; Elkeles, 1974) ; activité phospholipasiques (Greten, 1972). Les enzymes mises en jeu au cours de la lipomobilisation ne semblent pas interférer avec le dosage car l'inhibition par le chlorure de sodium est maintenue lorsque l'animal présente des signes de lipolyse intense.

Des activités monoglycéride-lipasiques (Gorin et Shafrir, 1964 ; Kupiecki, 1966 ; Heller et Steinberg, 1972 ; Katocks et al., 1972) et triglycéride-lipasiques (Mann et Tove, 1965) du tissu adipeux, peu inhibées par le fluorure de sodium et partiellement inhibées par le chlorure de sodium ont cependant été observées, mais elles n'étaient pas inhibées comme dans notre cas par l'absence de sérum dans le milieu. De plus, l'Intralipide activé par du sérum est un substrat très peu sensible aux activités monoglycéride-lipasiques (Biale et Shafrir, 1969 ; Fielding, 1972 ; Elkeles, 1974). La très faible hydrolyse du substrat, lorsqu'il n'est pas activé par du sérum, est une caractéristique de la L.P.L. (Korn, 1955).

L'activité lipolytique obtenue par la méthode I.V. est moins sensible à la nonactivation de l'Intralipide, ce qui est probablement dô à une activation partielle de celui-ci par le sérum contenu dans l'extrait brut (4 p. 100).

L'activité triglycéride-lipasique « hormonosensible » à pH acide (Loffler et Weiss, 1970) ne devrait pas se manifester au pH utilisé ici, ef celle agissant à pH neutre n'est pas présente dans les poudres acétoniques de tissu adipeux (Vaughan et al., 1964).

L'activité lipolytique observée est faible lorsque l'animal est dans un état physiologique de mobilisation des réserves corporelles. En effet, l'activité lipolytique est 5 fois plus faible en début de lactation qu'en fin de gestation (Chilliard et al., 1976) et nous avons observé, chez une chèvre ayant subi $36 \mathrm{~h}$ de jeûne, une activité multipliée par 15 (exprimée par rapport au poids sec dégraissé de tissu) après 6 jours de réali- 
mentation (Chilliard, non publié). Cefte activité lipolytique semble donc bien impliquée dans le dépôt des lipides corporels.

L'activité lipolytique observée par chacune des 3 méthodes semble donc due essentiellement à la L.P.L.

L'héparine joue un rôle important dans l'extraction de l'enzyme (fig. 2), conformément aux résultats rapportés par de Gasquet et Pequignot (1972) (méthode H.A.) et Robinson (1963) (méthode E.A.).

L'élution dans une incubation in vitro en absence d'héparine des deux tiers de l'activité obtenue avec le système complet, contrairement aux résultats classiquement observés (Cherkes et Gordon, 1959 ; Elkeles, 1974) pourrait s'expliquer par la présence de sérum (4 p. 100) et d'albumine (1,1 p. 100) dans le milieu. En effet, Stewart et Schotz (1973), Nilsson-Ehle (1974) et Kornhauser et Vaughan (1975) ont rapporté que le sérum permet une élution accrue d'activité L.P.L. à partir du tissu adipeux ou des adipocytes. Par ailleurs, Garfinkel et al. (1976) mentionnent un grand pouvoir d'élution de l'enzyme des adipocytes par certains lots d'albumine bovine (fraction V).

Le fissu adipeux de chèvre incubé in vitro en milieu hépariné libère donc de la L.P.L., contrairement à ce qui a été observé par Benson (1969) avec le tissu de vache laitière. Outre une différence liée à l'espèce, ces résultats divergents pourraient être dus aux conditions expérimentales dissemblables utilisées.

Les études de l'influence de l'héparine sur l'essai de l'activité L.P.L. extraite en absence d'héparine donnent des résultats variables et souvent contradictoires, selon les conditions expérimentales employées par les auteurs. Au cours de la présente investigation, seule l'activité lipolytique extraite par la méthode E.A. en absence d'héparine, est stimulée par celle-ci lors de l'incubation enzymatique. Ces extraits, non centrifugés, contiennent de fines particules de tissu en suspension sur lesquelles l'héparine pourrait agir en libérant l'enzyme au cours de l'incubation (Robinson, 1963), reproduisant ainsi son effet sur l'extraction.

Influence de la concentration en substrat.

Une inhibition de l'activité L.P.L. du tissu adipeux par l'excès de substrat (le rapport triglycérides/activateurs sanguins étant constant) a aussi été observée par Benson (1969) et Evans (1972). Par contre, Korn (1962) et Brady ef Higgins (1967) n'ont pas enregistré ce phénomène, mais les concentrations maximum en substrat employées par ces auteurs, relativement aux $\mathrm{Km}$ apparents observés, étaient généralement plus faibles. Cette inhibition entraîne une sous-estimation non négligeable de la vitesse maximum apparente calculée, lorsqu'on utilise une seule concentration en substrat pour estimer cette vitesse (fig. 3).

La valeur absolue du $\mathrm{Km}$ apparent enregistrée ci-dessus avec des extraits enzymatiques bruis doit être considérée avec prudence, d'autant plus que les valeurs du $\mathrm{Km}$ apparent présentent de grandes variations selon la nature du substrat employé (Korn, 1962 ; Boberg et Carlson, 1964 ; Brady et Higgins, 1967 ; de Pury et Collins, 1972 ; Fielding, 1973), et que la concentration effective en substrat pour l'enzyme dépend de l'aire de l'interface de l'émulsion (Benzonana et Desnuelle, 1965) qui est inconnue dans cette étude. Le $\mathrm{Km}$ apparent de l'activité L.P.L. du lait de chèvre 
(0,25 mM, pour le même substrat, Chilliard et Fehr, 1976) est cependant très voisin de celui du tissu adipeux de cette espèce $(0,19 \mathrm{mM})$.

Reproductibilité des différentes étapes de la mesure de l'activité L.P.L.

Les reproductibilités observées sont comparables à celles rapportées par d'autres auteurs (Benson, 1969 ; Boberg, 1970 ; Fruchart, 1972 ; Nilsson-Ehie, 1974), si l'on tient compte des faibles activités L.P.L. mesurées ici. La plus mauvaise reproductibilité de la méthode I.V. pourrait être due à des variations dans la finesse de coupe du tissu. De plus, cette méthode donne lieu à des concentrations en A.G.N.E. élevées et variables dans l'extrait enzymatique, probablement en raison d'une hydrolyse des triglycérides du tissu adipeux ou du sérum par la L.P.L., ou d'autres lipases, au cours de l'incubation préalable in vitro. Ceci contribue à diminuer la reproductibilité et oblige à multiplier les dosages.

Comparaison des activités obtenues par les trois méthodes.

Les résultats de la comparaison méthode I.V.-méthode E.A. sont en accord avec ceux de Elkeles (1974) sur tissu adipeux humain et de Brady et Higgins (1967) et Pykalisto et al. (1974) sur tissu adipeux de rat. Avec le tissu adipeux humain Nilson-Ehle (1974) et Pykalisto et al. (1975) rapportent une activité L.P.L. éluée en milieu hépariné plus forte que l'activité L.P.L. extraite des poudres acéto-éthérées. Toutefois, ces auteurs n'utilisent pas d'héparine avec les poudres acéto-éthérées ef n'emploient pas le même substrat, ni les mêmes conditions d'incubation in vitro que Elkeles (1974), qui elles-mêmes diffèrent des conditions utilisées dans cette étude.

Le faił d'obtenir des activités L.P.L. voisines par les trois méthodes ne signifie pas que celles-ci soient strictement équivalentes :

L'activité éluée par incubation in vitro représente probablement la fraction extra-adipocytaire de l'enzyme, localisée à la surface de la lumière des capillaires sanguins (Nilsson-Ehle et al., 1976). Par ailleurs, il se peut que les adipocytes qui forment le tissu sécrètent l'enzyme au cours de l'incubation, de pair avec une activation de celle-ci, qui peut aussi s'inactiver une fois libérée (Robinson et Wing, 1970 ; NilssonEhle, 1974 ; Stewart et Schotz, 1974 ; Kornhauser et Vaughan, 1975 ; Nilsson-Ehle et al., 1976). L'activité mesurée est donc la résultante, dans des conditions expérimentales données, de plusieurs phénomènes (Nilsson-Ehle, 1974).

L'activité L.P.L. obtenue par extraction de poudres acéto-éthérées du tissu devrait représenter la totalité de l'activité L.P.L. du tissu, intra et extraadipocytaire. L'utilisation d'une grande quantité de tampon ammoniacal hépariné (environ $13 \mathrm{ml} / \mathrm{mg}$ de poudres) devrait assurer une extraction presque quantitative de l'enzyme (Robinson, 1963) comme le suggèrent nos résultats. Toutefois, une certaine inactivation a été observée par Fielding (1968), et Okuda ef Fujii (1973) estiment que l'activité L.P.L. du tissu adipeux est fortement diminuée par l'extraction acéto-éthérée.

L'activité L.P.L. présente dans les homogénats aqueux se rapproche de celle éluée in vitro, puisque l'homogénéisation a lieu en milieu hépariné, ef de celle extraite des poudres acéto-éthérées, dans la mesure où l'homogénéisation permet de libérer l'enzyme intraadipocytaire. Cependant, une fraction de la L.P.L. pourrait s'adsorber sur les particules grasses, mises en suspension puis éliminées (Robinson, 1963 ; Gorin et Shafrir, 1964 ; Okuda et Fujii, 1973). 
Il est donc difficile de prévoir quelle méthode devrait donner une activité L.P.L. maximale, les résultats obtenus étant la résultante d'un grand nombre de facteurs liés aux conditions expérimentales utilisées el aux processus physiologiques tissulaires. II est cependant positif de constater de bonnes corrélations entre les trois méthodes d'extraction, d'un animal à l'autre ef pour des états physiologiques variés.

\section{Conclusion.}

Une activité lipoprotéine-lipasique est présente dans le tissu adipeux de chèvre. Des activités L.P.L. ont aussi été mises en évidence dans le sang veineux mammaire (Barry ef al.,1963) et dans le plasma post-héparine (Gill, 1970), ainsi que dans la mamelle (Chilliard, non publié) et dans le lait (Chilliard et Fehr, 1976) de cette espèce. L'activité L.P.L. du tissu adipeux épiploïque de la chèvre en fin de gestation et en début de lactation, exprimée par gramme du tissu, est du même ordre de grandeur que les activités observées dans les tissus adipeux de ratte (Otway et Robinson, 1968 ; Hamosh ef al., 1970) et de vache (Shirley et al., 1973) aux mêmes stades physiologiques.

Les interactions de l'enzyme du tissu adipeux de chèvre avec les activateurs sériques, la force ionique du milieu $(\mathrm{NaCl} 0,5 \mathrm{M})$, le fluorure de sodium, l'héparine, les méthodes d'extraction spécifiques de la L.P.L. (méthodes I.V. ef E.A.), ainsi que les variations physiologiques de son activité lipolytique (fin de gestation-début de lactation ; jeûne-réalimentation) permettent de penser que la L.P.L. du tissu adipeux de chèvre présente les mêmes caractéristiques que celle des autres mammifères.

Des trois méthodes employées pour mesurer l'activité L.P.L. du tissu adipeux de chèvre, l'incubation in vitro fournit les résultats les moins reproductibles. Cette méthode présente cependant l'avantage de permettre des éfudes métaboliques in vitro. Les méthodes par homogénéisation en milieu aqueux et par extraction des poudres acétoéthérées permettent d'obtenir des activités L.P.L. très voisines, tant en ce qui concerne les caractéristiques de l'activité enzymatique que la mesure absolue. Bien que la méthode E.A. soit considérée comme plus spécifique, nous pensons que des études supplémentaires seraient nécessaires pour mieux caractériser ces deux méthodes d'extraction. La méthode H.A. est par ailleurs plus rapide et moins onéreuse.

Accepté en aoû 1976.

Remerciements. Nous remercions Monsieur le Professeur H. Le Bars et Monsieur $H$. Brugère ; Monsieur G. Durand et Mademoiselle E. Penct ; Monsieur P. de Gasquet et Madame E. Pequignot-Planche; Messieurs J. Hervieu, A. Cucci, P. Bas ef D. Saurant pour les conseils ef l'assistance qu'ils nous ont fournis au cours de ce travail.

\section{Références}

ANTONIS A., 1965. Semiautomated method for the colorimetric determination of plasma free fatty acids. J. Lipid. Res., 6, 307-312.

BARRY J. M., BARTLEY W., LINZELL J. L., ROBINSON D. S., 1963. The uptake from the blood of triglyceride fatty acids of chylomicra and low density lipoproteins by the mammary gland of the goat. Biochem. J., 89, 6-11. 
BENSADOUN A., EHNHOLM C., STEINBERG D., BROWN W. V., 1974. Purification and characterization of lipoprotein lipase from pig adipose tissue. J. Biol. Chem., 249, 2220-2227.

BENZONANA G., DESNUELLE P., 1965. Etude cinétique de l'action de la lipase pancréatique sur des triglycérides en émulsion. Essai d'une enzymologie en milieu hétérogène. Biochim. Biophys. Acta, 105, 121-136.

BENSON J. D., 1969. Lipid metabolism in bovine liver and adipose tissue. Ph. D. thesis, Michigan State Univ., East Lansing, U. S. A.

BEZMAN A., FELTS J. M., HAVEL R. J., 1962. Relation between incorporation of triglyceride fatty acids and heparin released lipoprotein lipase from adipose tissue slices. J. Lipid. Res., 3, 427-431.

BIALE Y., SHAFRIR E., 1969. Lipolytic activity toward tri and monoglycerides in postheparin plasma. Clin. Chim. Acta., 23, 413-419.

BOBERG J., CARLSON L. A., 1964. Determination of heparin induced lipoprotein lipase activity in human plasma. Clin. Chim. Acta, 10, 420-427.

BOBERG J., 1970. Quantitative determination of heparin released lipoprotein lipase activity in human plasma. Lipids, 5, 452-456.

BRADY M., HIGGINS J. A., 1967. The properties of the lipoprotein lipases of rat heart, lung and adipose tissue. Biochim. Biophys. Acta, 137, 140-146.

CHERKES A., GORDON R. S., 1959. The liberation of lipoprotein lipase by heparin from adipose tissue incubated in vitro. J. Lipid. Res., 1, 97-101.

CHILLIARD Y., FEHR P. M., 1976. Activité lipolytique du lait de chèvre. I. Mise en évidence d'une activité lipoprotéine-lipasique Ann. Technol. agric. 25 (sous presse).

CHILLIARD Y., SAUVANT D., HERVIEU J., DORLEANS M., FEHR P. M., 1976. Activité lipoprotéine-lipasique et composition du tissu adipeux épiploïque en relation avec le métabolisme lipidique de la chèvre en fin de gestation et en début de lactation (à paraître).

COHEN P. P., 1949. Suspending media for animal tissues. In UMBREIT W. W., BURRIS R. H., STAUFFER J. F., Manometric techniques and tissue metabolism, 118-119. Burgess Publishing \& Co, Indiapolis, U. S. A.

DE GASQUET P., PEQUIGNOT E., 1972. Lipoprotein lipase activities in adipose tissues, heart and diaphragm of the genetically obese mouse (ob/ob). Biochem. J., 127, 445-447.

DE PURY G. G., COLLINS F. D., 1972. Very low density lipoproteins and lipoprotein lipase in serum of rats deficient in essential fatty acids. J. Lipid. Res., 13, 268-275.

DOLE V. P., 1956. A relation between non esterified fatty acids in plasma and metabolism of glucose. J. Clin. Invest., 35, 150-154.

EISENTHAL R., CORNISH-BOWDEN A., 1974. The direct linear plot. A new graphical procedure for estimating enzyme kinetic parameters. Biochem J., 139, 715-720.

ELKELES R. S., 1974. Lipoprotein lipase in human adipose tissue. Clin. Sci. Mol. Med., 46, 753-762.

EVANS A. J., 1972. Lipoprotein lipase activity in adipose tissues of the domestic duck : the effect of age, sex, and nutritional state. Int. J. Biochem., 3, 199-206.

FIELDING C. H., 1968. Inactivation of lipoprotein lipase in buffered saline solutions. Biochim. Biophys. Acta, 159, 94-102.

FIELDING C. J., 1972. Further characterisation of lipoprotein lipase and hepatic post-heparin lipase from rat plasma. Biochim. Biophys. Acta, 280, 569-578.

FIELDING C. J., 1973. Kinetics of lipoprotein lipase activity : effects of the substrate apoprotein on reaction velocity. Biochim. Biophys. Acta, 316, 66-75.

FRUCHART J. C., 1972. Contribution à l'étude de la lipoprotéine-lipase du plasma post-héparine et des tissus. Thèse doct. Etat. Pharm. Lille, France.

GARFINKEL A. S., BAKER N., SCHOTZ C. M., 1967. Relationship of lipoprotein lipase activity to triglyceride uptake in adipose tissue. J. Lipid. Res., 8, 274-280. 
GARFINKEL A. S., NILSSON-EHLE P., SCHOTZ M. C., 1976. Regulation of lipoprotein lipase. Induction by insulin. Biochim. Biophys. Acia, 424, 264-273.

GILL R. A., 1970. Studies on lipid metabolism in ruminants. Ph. D. Thesis. Univ. Wisconsin, U. S. A.

GORIN E., SHAFRIR E., 1964. Lipolytic activity in adipose tissue homogenate toward tri-, di-, and monoglyceride substrates. Biochim. Biophys. Acta, 84, 24-34.

GRETEN H., 1972. Post-heparin plasma phospholipases in normal and patients with hyperlipoproteinemia. Klin. Wschr., 50, 39-41.

HAMOSH M., CLARY T. R., CHERNICK S. S., SCOW R. O., 1970. Lipoprotein lipase activity of adipose and mammary tissue and plasma triglyceride in pregnant and lactating rats. Biochim. Biophys. Acta, 210, 473-482.

HAUGEBAK C. D., HEDRICK H. B., ASPLUND J. M., 1974. Relationship between extramuscular adipose tissue lipoprotein lipase activity and intramuscular lipid deposition in fattening lambs. J. anim. Sci., 39, 1026-1031.

HELLER R. A., STEINBERG D., 1972. Partial glyceridase activity of a protein-kinase activatable triglyceride lipase from rat adipose tissue. Biochim. Biophys. Acto, 270, 65-73.

KATOCS A. S. Jr., GNEWUCH C. T., LECH J. J., CALVERT D. N., 1972. Further studies on monoolein lipase from rat adipose tissue. Biochim. Biophys. Acta, 270, 209-217.

KORN E. D., 1955. Clearing factor lipase, a heparin-activated lipoprotein lipase. II. Substrate specificity and activation of coconut oil. J. Biol. Chem., 215, 15-26.

KORN E. D., 1959. The assay of lipoprotein lipase in vivo et in vitro. In GLUCK D. Methods of Biochemical Analysis, Vol. 7, 145-192. Intersciences Publishers, N. Y., London.

KORN E. D., 1962. The kinetics of the inhibition of lipoprotein lipase by polyanions and polycations. J. Biol. Chem., 237, 3423-3429.

KORN E. D., QUIGLEY T. W., 1955. Studies on lipoprotein lipese of rat heart and adipose tissue. Biochim. Biophys. Acta, 18, 143-145.

KORNHAUSER D. M., VAUGHAN M., 1975. Release of lipoprotein lipase from fat cells in vitro. Biochim. Biophys. Acfa, 380, 97-105.

KUPIECKI F. P., 1966. Partial purification of monoglyceride lipase from adipose tissue. J. Lipid. Res., 7, 230-235.

LOFFLER G., WEISS L., 1970. Lipase activities in adipose tissue. In JEANRENAUD B., HEPP D. Adipose tissue. Regulation and metabolic functions, 32-36. George Thieme Verlag, Stuttgart ; Acad. Press, N. Y., Londres.

MANN J. T., TOVE S. B., 1966. Purification and properties of a lipase from rat adipose tissue. J. Biol. Chem., 241, 3595-3599.

NILSSON-EHLE P., 1974. Human lipoprotein lipase activity : comparison of assay methods. Clin. Chim. Acta, 54, 283-291.

NILSSON-EHLE P., GARFINKEL A. S., SCHOTZ M. C., 1976. Intra- and extracellular forms of lipoprotein lipase in adipose tissue. Biochim. Biophys. Acta, 431, 147-156.

NOVAK M., 1965. Colorimetric ultramicro method for the determination of free fatty acids. J. Lipid Res., 6, 431-433.

OKUDA H., FUJII S., 1973. Studies on hormone-sensitive lipase and lipoprotein lipase in adipose tissue. J. Biochim., 73, 1195-1203.

OTWAY S., ROBINSON D. S., 1968. The significance of changes in tissue clearing-factor lipase activity in relation to the lipaemia of pregnancy. Biochem. J., 106, 677-682.

PYKALISTO O. J., VOGEL W. C., BIERMAN E. L., 1974. The tissue distribution of triacylglycerol lipase, monoacylglycerol lipase and phospholipase $A$ in fed and fasted rats. Biochim. Biophys. Acta, 369, 254-263.

PYKALISTO O. J., SMITH P. H., BRUNZELL J. D., 1975. Human adipose tissue lipoprofein lipase : comparison of assay methods and expressions of activity. Proc. Soc. Exp. Biol. Med., 148, 297. 300 . 
RIZACK M. A., 1961. An epinephrine sensitive lipolytic activity in adipose tissue. J. Biol. Chem., 236, 657-662.

ROBINSON D. S., 1963. Clearing factor lipase and its action in the transport of fatty acids between the blood and the tissues. Adv. Lipid. Res., 1, 133-181.

ROBINSON D. S., WING D. R., 1970. Regulation of adipose tissue clearing factor lipase activity. Horm. Metab. Res., 2, suppl. 2, 41-46.

SCOW R. O., HAMOSH M., BLANCHETTE-MACKIE E. J., EVANS A. J., 1972. Uptake of blood triglyceride by various tissues. Lipids, 7, 497-505.

SHIRLEY J.E., EMERY R. S., CONVEY E. M., OXENDER W. D., 1973. Enzymic changes in bovine adipose and mammary tissue, serum and mammary tissue hormonal changes with initiation of lactation. J. Dairy Sci., 56, 569-574.

SNEDECOR G. W., COCHRAN W. G., 1957. Statistical methods. lowa state university press.

STEWART J. E., SCHOTZ M. C., 1973. Stimulation of release of lipoprotein lipase from isolated fat cells by rat serum. Nature New Biol., 244, 250-251.

STEWART J. E., SCHOTZ M. C., 1974. Release of lipoprotein lipase activity from isolated fat cells. II. Effect of heparin. J. Biol. Chem., 249, 904-907.

STRAND O., VAUGHAN M., STEINBERG D., 1964. Rat adipose tissue lipases : hormone-sensitive lipase activity against triglycerides compared with activity against lower glycerides. J. Lipid. Res., 5, 554-562.

VAUGHAN M., BERGER J. E., STEINBERG D., 1964. Hormone-sensitive lipase and monoglyceride lipase activities in adipose tissue. J. Biol. Chem., 239, 401-409. 